CVIA

REVIEW ARTICLE

pISSN 2508-707X / eISSN 2508-7088 https://doi.org/10.22468/cvia.2017.00115 CVIA 2017;1(4):222-230

\title{
Postoperative Complications after Surgical Aortic Valve Replacement
}

\author{
Hyun Jung Koo', Han Na Lee ${ }^{1,2}$, Tran ThiXuan Anh³, \\ Joon-Won Kang ${ }^{1}$, Dong Hyun Yang ${ }^{1}$, Jae-Kwan Song ${ }^{4}$, \\ Duk-Hyun Kang ${ }^{4}$, Jong-Min Song ${ }^{4}$, Jae Won Lee, \\ Cheol Hyun Chung ${ }^{5}$, Suk-Jung Choo ${ }^{5}$, Tae-Hwan Lim ${ }^{1}$ \\ 'Department of Radiology and Research Institute of Radiology, Cardiac Imaging Center, \\ Asan Medical Center, University of Ulsan College of Medicine, Seoul, Korea \\ 2Department of Radiology, Kyung Hee University Hospital at Gangdong, Seoul, Korea \\ ${ }^{3}$ Department of Cardiology, University Medical Center, Ho Chi Minh, Vietnam \\ ${ }^{4}$ Division of Cardiology, Department of Internal Medicine, Asan Medical Center, \\ University of Ulsan College of Medicine, Seoul, Korea \\ ${ }^{5}$ Department of Cardiothoracic Surgery, Asan Medical Center, Seoul, Korea
}

Received: August 23, 2017

Revised: September 20, 2017

Accepted: September 25, 2017

Corresponding author

Joon-Won Kang, MD

Department of Radiology and

Research Institute of Radiology,

Cardiac Imaging Center,

Asan Medical Center, University of Ulsan

College of Medicine,

88 Olympic-ro-43-gil, Songpa-gu,

Seoul 05505, Korea

Tel: 82-2-3010-3997

Fax: 82-2-476-0090

E-mail: jwonkang@amc.seoul.kr

\begin{abstract}
Aortic valve replacement is one of the most common major cardiac surgeries worldwide. Despite improvements in surgical techniques, postoperative complications after aortic valve surgery remain a source of substantial morbidity and mortality. Although transthoracic echocardiography and transesophageal echocardiography have been used for routine surveillance of prosthetic heart valves, computed tomography (CT) has played an important role with echocardiography in the diagnosis, treatment, and follow-up of patients after valve replacement. CT has the advantage of evaluating the extent of disease, such as paravalvular dehiscence, abscess, and pseudoaneurysm, in aortic prosthetic valves. In this article, we review the various image findings of postoperative complications after aortic valve replacement detected on $\mathrm{CT}$.
\end{abstract}

Key words Aortic valve surgery - Aortic valve replacement - Computed tomography . Postoperative $\cdot$ Complications.

\section{INTRODUCTION}

The prevalence of aortic valve disease is increasing with the aging of the population, with a reported incidence of approximately $3-4 \%$ in the adult population $[1,2]$. Aortic valve disease is the most common valvular disease requiring valve replacement. Although transcatheter aortic valve implantation (TAVI) shows good outcome for selected patients, aortic valve replacement (AVR) remains an equivalent treatment for patients with aortic valve stenosis or aortic valve regurgitation [2].

Despite much progress in surgical procedures and prosthetic valve design, complications after AVR significantly affect patient mortality; the reported 30-day and 1-year mortality rates are $2.1 \%$ and $4.9 \%$, respectively [3]. The incidence of various complications after AVR is affected by valve type (mechanical vs. bioprosthetic), valve position, and clinical risk factors. Compli-

(c) This is an Open Access article distributed under the terms of the Creative Commons Attribution Non-Commercial License (http://creativecommons.org/licenses/bync/4.0) which permits unrestricted non-commercial use, distribution, and reproduction in any medium, provided the original work is properly cited. cations of AVR include paravalvular aortic regurgitation, dehiscence, infective endocarditis, aortic dissection, and hemolysis (Table 1).

Routine screening with echocardiography after aortic valve surgery is essential to detect postoperative or postprocedural complications. Echocardiography has several strengths, including ready availability, bedside examination suitability, and low cost. It provides information not only on the motion of the prosthetic valve, but also on functional information using Doppler imaging parameters, such as pressure gradients and flow velocity through the valve. However, echocardiography is an operator-dependent modality, and lesions can be obscured by prosthetic valve artifacts. To obtain additive information, computed tomography (CT) examination has been widely used. Cardiac CT imaging is a rapid, noninvasive, and accurate technique that enables clinicians to obtain a quick diagnosis and assists in treatment planning. However, CT does not provide real-time imaging, and beta-blocker medication is often necessary to reduce the heart rate during the $\mathrm{CT}$ acquisition due to the limited tem- 
Table 1. Complications after aortic valve replacement

\begin{tabular}{lc}
\hline \multicolumn{1}{c}{ Complications } & Rate (\%) \\
\hline Early & \\
Paravalvular aortic regurgitation & 30-day rate: 0.9 \\
& 1-year rate: 0.5 \\
Aortic dissection & 0.6 \\
Coronary artery occlusion (stenosis) & $0.3-5$ \\
Stroke & $7-17$ \\
Mediastinitis & $0.9-20^{*}$ \\
Late & \\
Infective endocarditis & $1-6$ \\
Pannus formation & $\mathrm{NA}$ \\
Sternal osteomyelitis & $1-3^{*}$ \\
Paravalvular aortic regurgitation & $0.1-1.3$ \\
Hemolysis & $5-15$ \\
\hline
\end{tabular}

${ }^{*}$ rates obtained from patients who underwent heart surgery, not only aortic valve surgery. NA: not available

poral resolution of CT. Electrocardiogram (ECG)-gating should be performed to obtain optimal images and reduce misinterpretation artifacts. Although we discuss the usefulness of CT to evaluate complications after AVR in this review, echocardiography and CT are complementary imaging modalities and are both necessary for optimal cardiac evaluation. The purpose of this review is to provide insights into complications after AVR for both clinicians and radiologists and to illustrate CT findings of postoperative complications.

\section{SPATIAL AND TEMPORAL RESOLUTION OF ECHOCARDIOGRAPHY AND CARDIAC CT}

Echocardiography is a first-line tool for non-invasive study of cardiac diseases due to its high temporal and special resolution, as well as its low cost, portability, lack of radiation, and ability to evaluate anatomic and functional information [4]. Spatial resolution better than $100 \mu \mathrm{m}$ and temporal resolution better than 600 frames/sec are now available for practical applications. Moreover, real-time, 3D color Doppler imaging can provide quantifying blood flow in the heart. However, echocardiography has several pitfalls, such as a poor sonic window in postoperative (sternotomy or mechanical valves) or obese patients and suboptimal evaluation of aorta and pericardial diseases [5]. Consequently, many patients undergo additional cardiac CT examination to evaluate cardiac lesions.

Although CT does not demonstrate real-time images, recent advancement of $\mathrm{CT}$ equipment and image processing methods have provided significant improvements in temporal resolution. Because of the limitation of temporal resolution on cardiac $\mathrm{CT}$, beta-blocker medication is often used to reduce heart rate during the $\mathrm{CT}$ acquisition. Also, considering that the spatial resolution of CT is reached at $0.3-0.4 \mathrm{~mm}$, nitroglycerin is sometimes given to patients to visualize small coronary arteries. The ability of cardiac imaging techniques relates to capture image detail (spatial resolution) during the shortest time expressed as temporal resolution (milliseconds). Fast gantry rotation speed allows capture of a large amount of data, which are necessary to evaluate the regional wall motion of the heart in a single cardiac cycle. Because an image can be reconstructed using $180^{\circ}$ of CT acquisition data, the temporal resolution would be $250 \mathrm{~ms}$ in a scanner with $500 \mathrm{~ms}$ rotation speed.

\section{CT PROTOCOL}

The postoperative heart CT protocol is similar to that of routine coronary CT angiography, but it can be modified to assess the sternotomy site, valve position and motion, and periaortic/ paraaortic complications [5]. We have routinely used retrospective ECG-gated CT to avoid motion artifacts and to achieve adequate image quality for the evaluation of coronary arteries and reconstruction of valve images. Evaluation should include the location and multiphase motion of the valve, potential thrombus (acute stage in the setting of inadequate anticoagulation) and thickening, and calcification and pannus formation around the valve. Coronary arteries are also evaluated not only for potential atheroma, but also for iatrogenic obstruction due to valve implant. Paravalvular structures including the aortic root and left ventricular outflow tract (LVOT) are assessed to determine whether there is any cause of obstruction. Immediate mediastinal complications, such as chest wall or sternal dehiscence, mediastinitis with or without complicated fluid, and source of hemothorax or postoperative bleeding, are also evaluated. In patients with suspected mediastinitis or an abscess, we perform additional 1-min delayed CT scans after obtaining routine coronary CT scans.

\section{Paravalvular aortic regurgitation}

Paravalvular aortic regurgitation is a potentially serious complication of AVR. It is the regurgitation of blood flow between the prosthetic ring and native annulus due to incomplete sealing. The reported incidence of paravalvular aortic regurgitation is $2-10 \%$ after valve replacement [6,7]. Most paravalvular aortic regurgitation cases are trivial or mild asymptomatic regurgitations that are incidentally detected on intraoperative transesophageal echocardiography (TEE) or postoperative transthoracic echocardiography (TTE) [8]. However, approximately $1-5 \%$ of patients progress to severe paravalvular aortic regurgitation, which occurs more frequently in the mitral valve than in the aortic valve $[9,10]$.

Abnormal pressure or traction forces on the prosthesis can 
cause incomplete sealing of the prosthetic valve after surgery [11]. The known risk factors for prosthetic valve dehiscence include bacterial endocarditis, aneurysm of the ascending aorta, and diffuse calcification of the native aortic valve. In addition, infection, suturing technique, and size and shape of the prosthesis are known to affect the risk of leaking [12,13]. The most common cause of dehiscence is bacterial endocarditis [14]. Early development of paravalvular aortic regurgitation can be associated with surgical technique and prostheses, and late development of paravalvular aortic regurgitation is mainly caused by dehiscence related to infective endocarditis [13]. With time, the inflammation extends from the prosthetic valve into the paravalvular tissue, leading to tissue destruction and necrosis. Without prompt diagnosis and proper treatment, destruction of the annulus leads to valvular dehiscence and paravalvular aortic regurgitation, which are serious complications [15]. The clinical manifestations of paravalvular aortic regurgitation depend on the severity of regurgitation, ranging from asymptomatic cases to congestive heart failure and hemolytic anemia. Paravalvular aortic regurgitation should be suspected in a patient with a new heart murmur [16].

Echocardiography is regarded as the gold standard modality to evaluate paravalvular aortic regurgitation. Due to acoustic artifacts caused by the prosthetic valve, TEE, rather than TTE, is used to assess the site and severity of paravalvular leakage. Threedimensional TEE has better diagnostic accuracy than two-dimensional TEE in patients with multiple defects [13]. Although cardiac CT cannot directly visualize regurgitation flow, it can accurately demonstrate the site and morphology of the leaks using multiplanar reconstruction and volume rendering techniques (Fig. 1) and can guide percutaneous repair to avoid repeat valve surgery $[17,18]$. By three-dimensional reconstruction, we can accurately determine the size, location, and shape of the dehiscence to facilitate surgical planning [19]. On CT images, dehis- cence is seen as a gap between the aortic annulus and margin of the prosthetic valve through which contrast material flows from the left ventricular chamber into the aortic root (Fig. 2). It is well visualized on LVOT multiplanar reformatted images $[12,20]$. Surgery has been the traditional treatment for symptomatic paravalvular aortic regurgitation; however, percutaneous transcatheter closure has been performed with technical success rates of $77-86 \%[21,22]$.

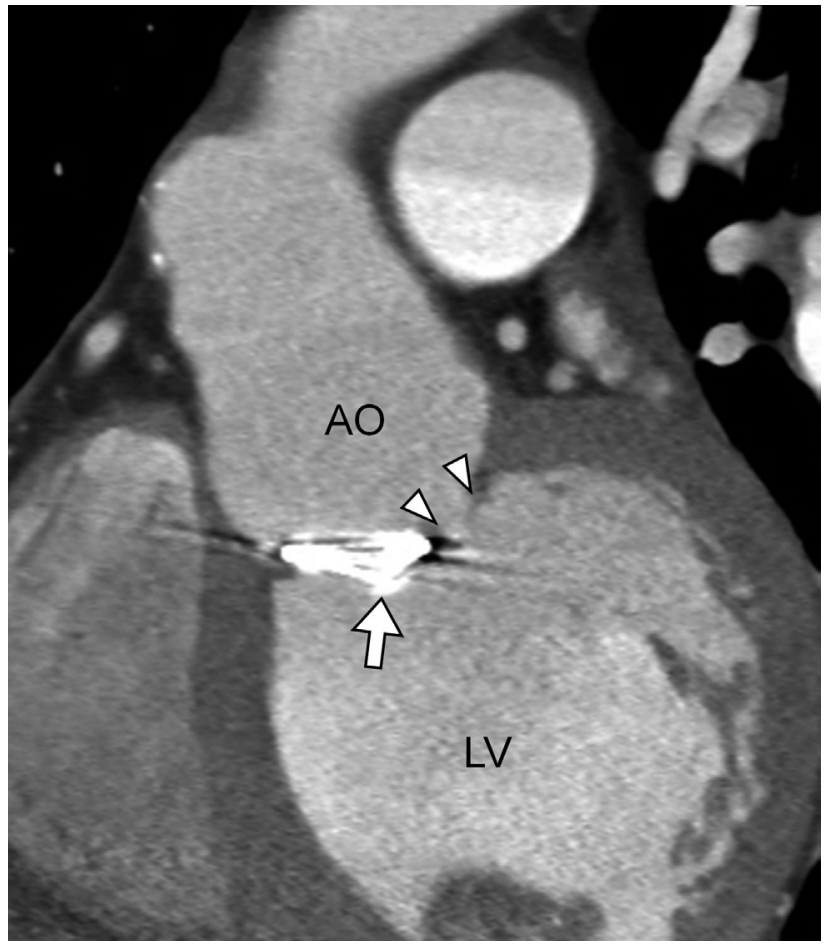

Fig. 2. A 43-year-old man who underwent surgical aortic valve replacement 9 years previously developed intermittent chest pain and visited the emergency department. On computed tomography, a large defect (arrowheads) is visible on the left side of the prosthetic valve (white arrow), indicating prosthetic valve dehiscence. AO: aorta, LV: left ventricle.
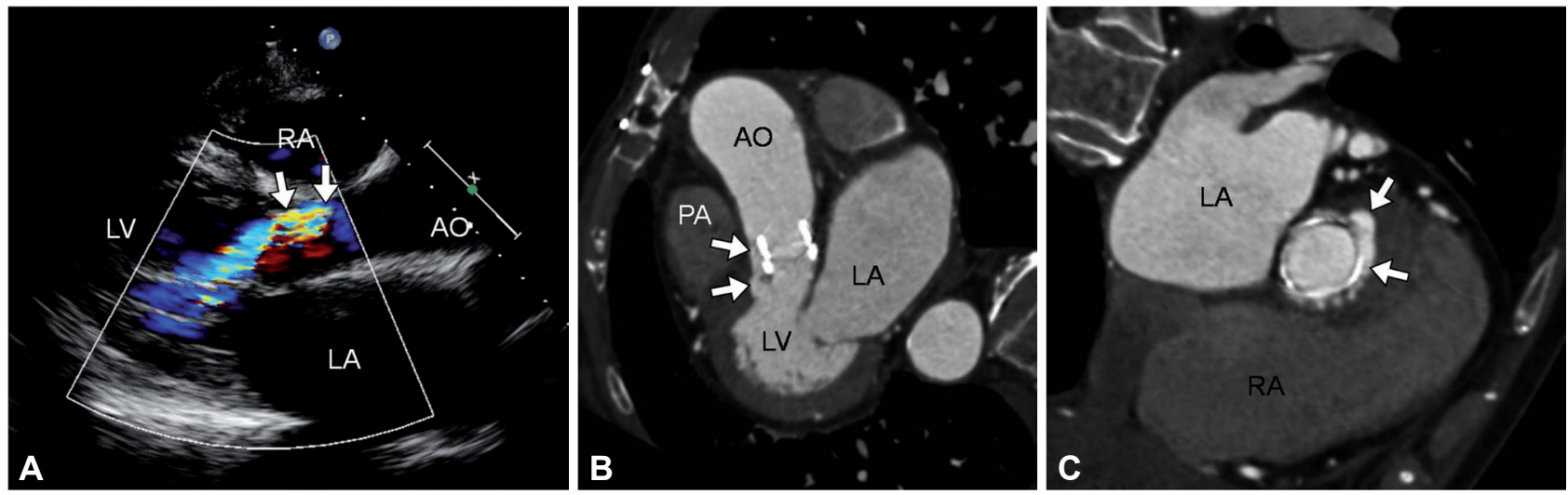

Fig. 1. An 88-year-old man who presented with dyspnea after repeat aortic valve replacement with a prosthetic tissue valve 3 months earlier due to severe aortic stenosis with infective endocarditis. (A) On postoperative echocardiography, moderate paravalvular leakage (arrows) projecting to the LV chamber during the diastolic phase is noted. (B and C) On computed tomography, a paravalvular defect (arrows) is noted just below the left coronary sinus. AO: aorta, LA: left atrium, LV: left ventricle, RA: right atrium, PA: pulmonary artery. 


\section{Thrombosis and pannus formation}

Prosthetic valve obstruction should be suspected in patients with increased transvalvular gradient following AVR or new symptoms of valve obstruction, such as dyspnea or heart failure. The most common causes of prosthetic valve obstruction are thrombosis and pannus formation $[23,24]$.

The reported prevalence of symptomatic obstructive thrombosis in mechanical valves ranges from $0.3 \%$ to $1.3 \%$, but subclinical (obstructive or nonobstructive) thrombosis is found in as many as $10 \%$ of cases [25]. The most important cause of prosthetic valve thrombosis is inadequate anticoagulation, which is more common with mechanical valves, but thrombosis can occur with bioprostheses long after implantation [23,26,27].

Pannus formation is a chronic complication after AVR, caused by fibrous tissue ingrowth around the prosthetic valve. The reported incidence of pannus formation is $0.2-4.5 \%$ [28,29]. In a surgical study, the incidence of pannus formation in combination with thrombus was $12 \%$ among 112 obstructed mechanical valves [28].

It is important to distinguish between thrombosis and pannus formation as the cause of valve occlusion. Thromboses can be treated with thrombolysis, whereas pannus formation requires surgical intervention. Although TTE and TEE are useful modalities for evaluating the severity of valve obstruction, they are less suitable for aortic valve obstruction because of acoustic shadowing, particularly if the mass is located on the anterior side of the aortic prosthetic valve [30,31].

Multidetector CT is useful to distinguish thrombus from
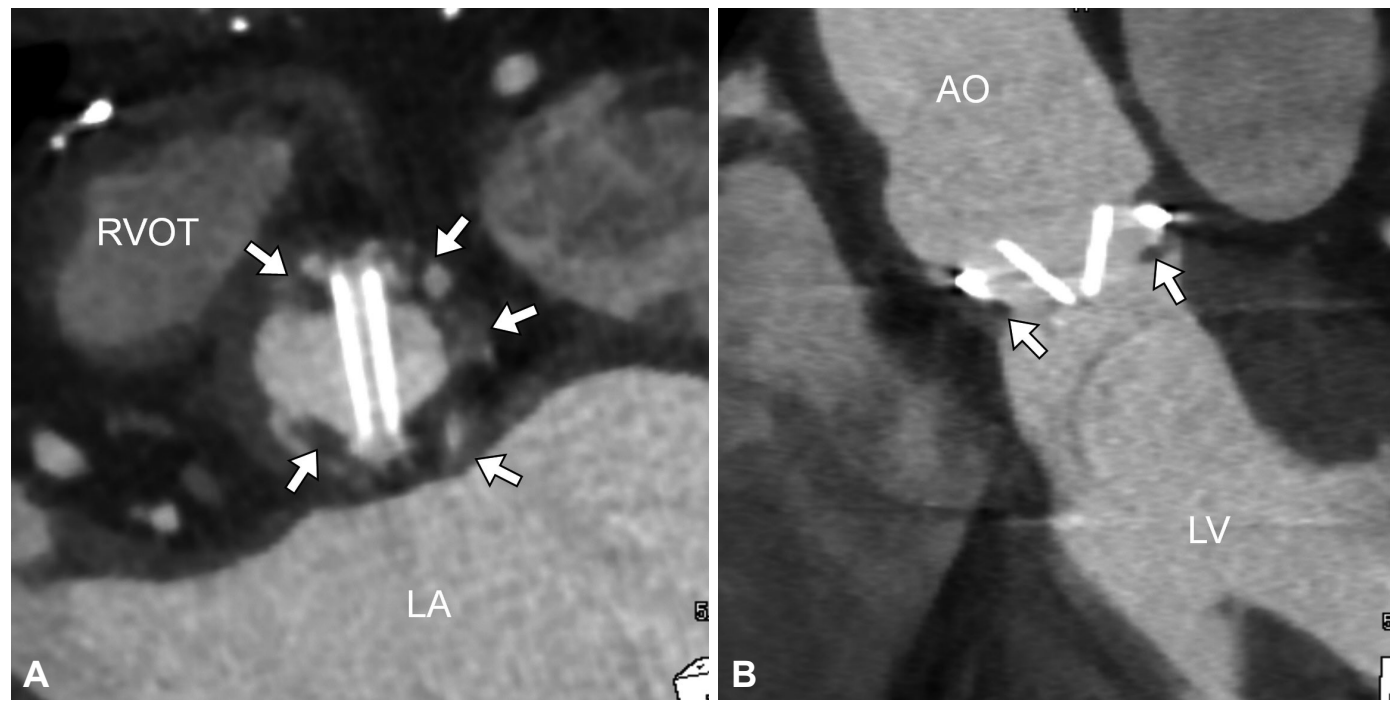

Fig. 3. Four years after surgical aortic valve replacement, a 49-year-old woman developed dyspnea. (A) On an aortic valve in-plane view, subvalvular tissue ingrowth (arrows) below the prosthetic aortic valve is noted. (B) A sagittal image of the left ventricular outflow tract shows the lesion in the subvalvular area (arrows). The lesion is showing 160-220 Hounsfield units on computed tomography, suggesting pannus formation. Transvalvular peak velocity $(3.4 \mathrm{~m} / \mathrm{s})$ and pressure gradient $(45 / 25 \mathrm{~mm} \mathrm{Hg})$ were high on echocardiography. AO: ascending aorta, LA: left atrium, LV: left ventricle, RVOT: right ventricular outflow tract.
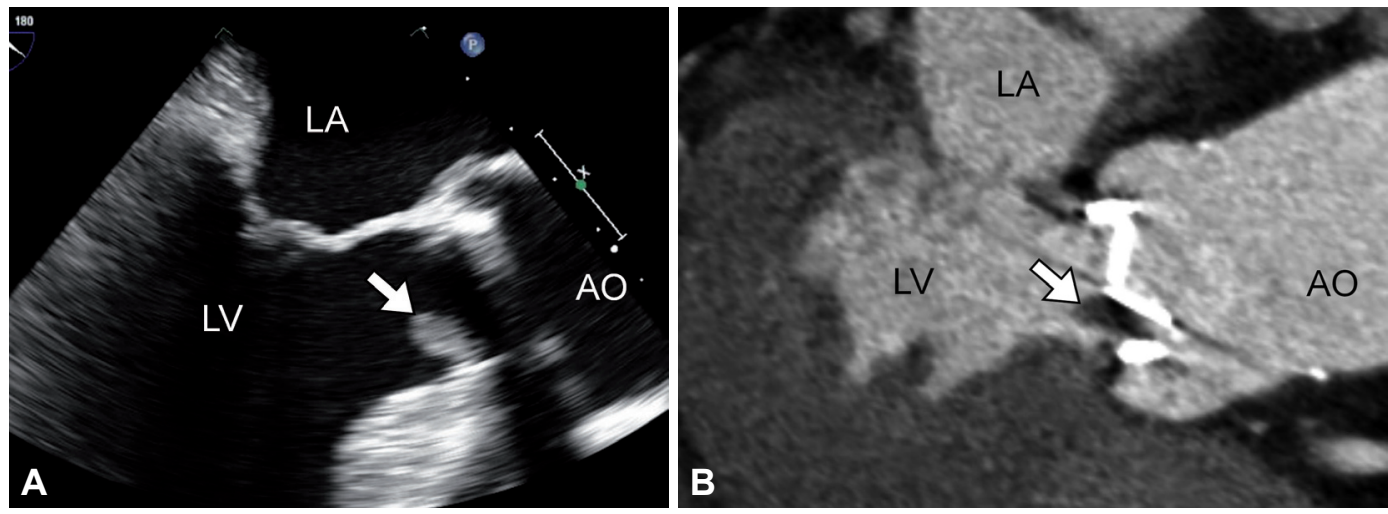

Fig. 4. A 65-year-old man who underwent surgical aortic valve replacement 9 years previously developed chest discomfort. The INR was 0.94 at the time of admission. (A) On echocardiography, a low-echoic movable lesion (white arrow) is visible with left prosthetic leaflet dysfunction. (B) The lesion (white arrow) shows low attenuation (52 Hounsfield units) on computed tomography and was suspected to be a thrombus. After adjustment for optimal anticoagulation, the INR increased to 2.3, and the lesion was no longer visible on follow-up echocardiography after 3 months (not shown). AO: aorta, LA: left atrium, LV: left ventricle, INR: international normalized ratio. 
pannus formation in prosthetic valves. Hounsfield unit of the lesions can be used to distinguish thromboses from panni, as panni tend to show higher attenuation. Gunduz et al. [32] reported that thromboses could be distinguished from panni with high sensitivity (87.5\%) and specificity (95.5\%) using a cut-off point of 145 Hounsfield units. A pannus is shown as a soft tissue attenuated lesion with a circular anatomical configuration along the subvalvular area to the valve ring (Fig. 3). In contrast, a thrombus is shown as a low attenuated lesion with irregular anatomy directly attached to leaflets and hinge points and causing restriction of leaflet motion [31,33-35] (Fig. 4).

\section{Sternal osteomyelitis and mediastinitis}

Sternal osteomyelitis occurs in $1-3 \%$ of patients who undergo sternotomy for heart surgery [36,37]. This condition can cause poststernotomy mediastinitis, which is a severe complication with a mortality rate of approximately $29 \%$ [37]. Its risk factors include obesity, diabetes, chronic obstructive pulmonary disease, chronic cough, steroid therapy, and internal mammary artery grafts [38]. Common CT findings in sternal osteomyelitis include bone destruction with demineralization and dehiscence [39]. Because aseptic hematoma or fluid collection in the mediastinum can be seen 2-3 weeks after sternotomy, it is not always easy to differentiate normal findings from those of infectious mediastinitis and abscess formation [40]. If mediastinal fluid collection with air persists for 2 weeks after operation, with redness and discharge from the sternotomy site, infectious mediastinitis should be suspected [41]. On CT images, mediastinal fluid collection with a thick enhancing peripheral rim, soft tissue edema, and sternal closure defects are suggestive of mediastinitis [42] (Fig. 5).

\section{Infective endocarditis}

The incidence of prosthetic valve endocarditis (PVE) is not especially low: it affects $1-6 \%$ of patients [43]. Although new diagnostic and therapeutic strategies have been introduced, high mortality rates of 19-40\% from aortic PVE have been reported $[15,44,45]$. PVE with onset within 2 months after surgery is classified as early PVE, while PVE that develops 12 months or more after surgery is late PVE. PVE occurring 2-12 months after surgery is classified as intermediate PVE $[46,47]$. Aortic PVE usually originates at the sewing cuff or at thrombi near the sewing ring [48]. Progression of the infection into the perivalvular tissue and abscess formation are more likely to occur with PVE than with native endocarditis [15,43] (Fig. 6). Abscesses, pseudoaneurysm formation, and valve dehiscence are complications of PVE that can occur in approximately $60 \%$ of patients $[12,47]$.

TTE and TEE are performed as part of routine surveillance in order to monitor complications in patients with AVR. How-

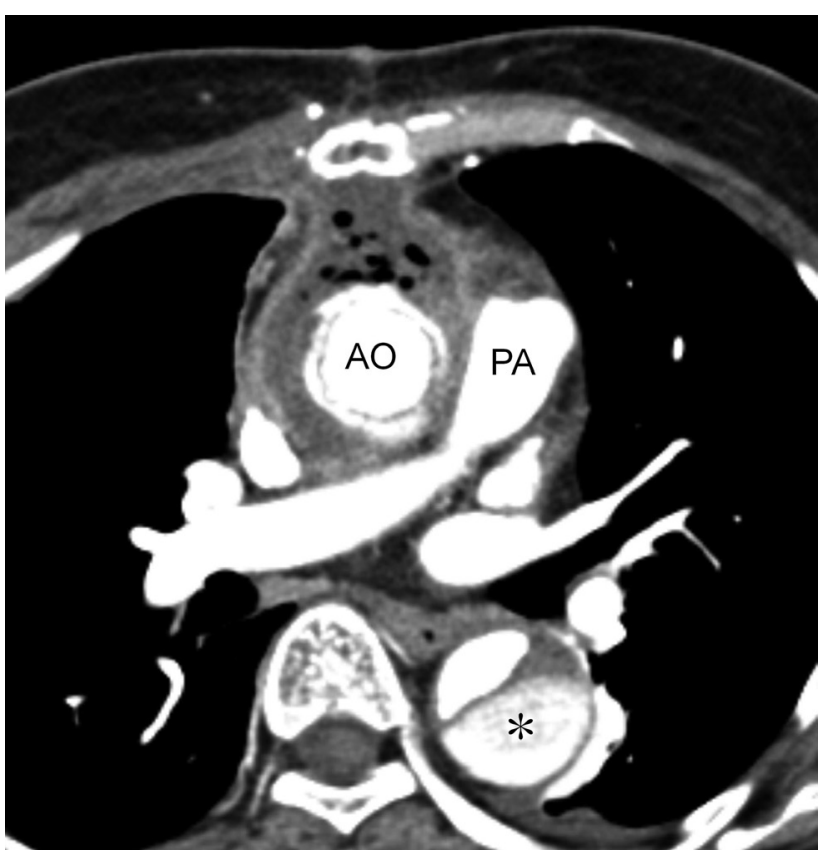

Fig. 5. A 75-year-old woman with severe aortic stenosis and aortic dissection managed by aortic valve replacement and ascending aorta and hemiarch replacement. After 17 days, she developed chest pain and fever. On computed tomography, a small amount of fluid with peripheral thick enhancement and multiple air densities suggesting mediastinitis with complicated fluid collection are visible in the anterior mediastinum and around the ascending aorta. Remaining dissection with preserved false lumen flow (asterisk) is noted in the descending thoracic aorta. The patient recovered after intensive antibiotic treatment. AO: aorta, PA: pulmonary artery.

ever, in patients with prosthetic valves, TTE and TEE play a limited role in the diagnosis of PVE compared with native valve endocarditis. TTE has a reported sensitivity of $36 \%$ and a specificity of $69 \%$; TEE has a reported sensitivity of $82 \%$ and a specificity of $88 \%[15,47,49]$.

Cardiac CT has an emerging role as a complementary diagnostic modality for PVE. The sensitivities of CT and TEE to detect vegetation are not significantly different for surgically confirmed infective endocarditis (100\% in TEE and 90.9-96\% in CT), and the perivalvular extent of abscess/pseudoaneurysms is more clearly demonstrable on CT than on TEE [50,51]. CT can provide a more accurate visualization of the anatomy to evaluate the extent and location of disease [52].

\section{Embolic events}

Thromboembolism can be caused by a thrombus on a prosthetic valve. Left side valve (mitral and aortic) thrombus can cause systemic embolic events such as stroke and myocardial infarction. The incidence of thromboembolism during the first 3 months after prosthetic valve replacement is reported to be approximately $3.6-6 \%$, which is significantly higher than the incidence after that period $[53,54]$. The long-term incidence of systemic embolization is approximately $0.7-1.0 \%$ in patients 

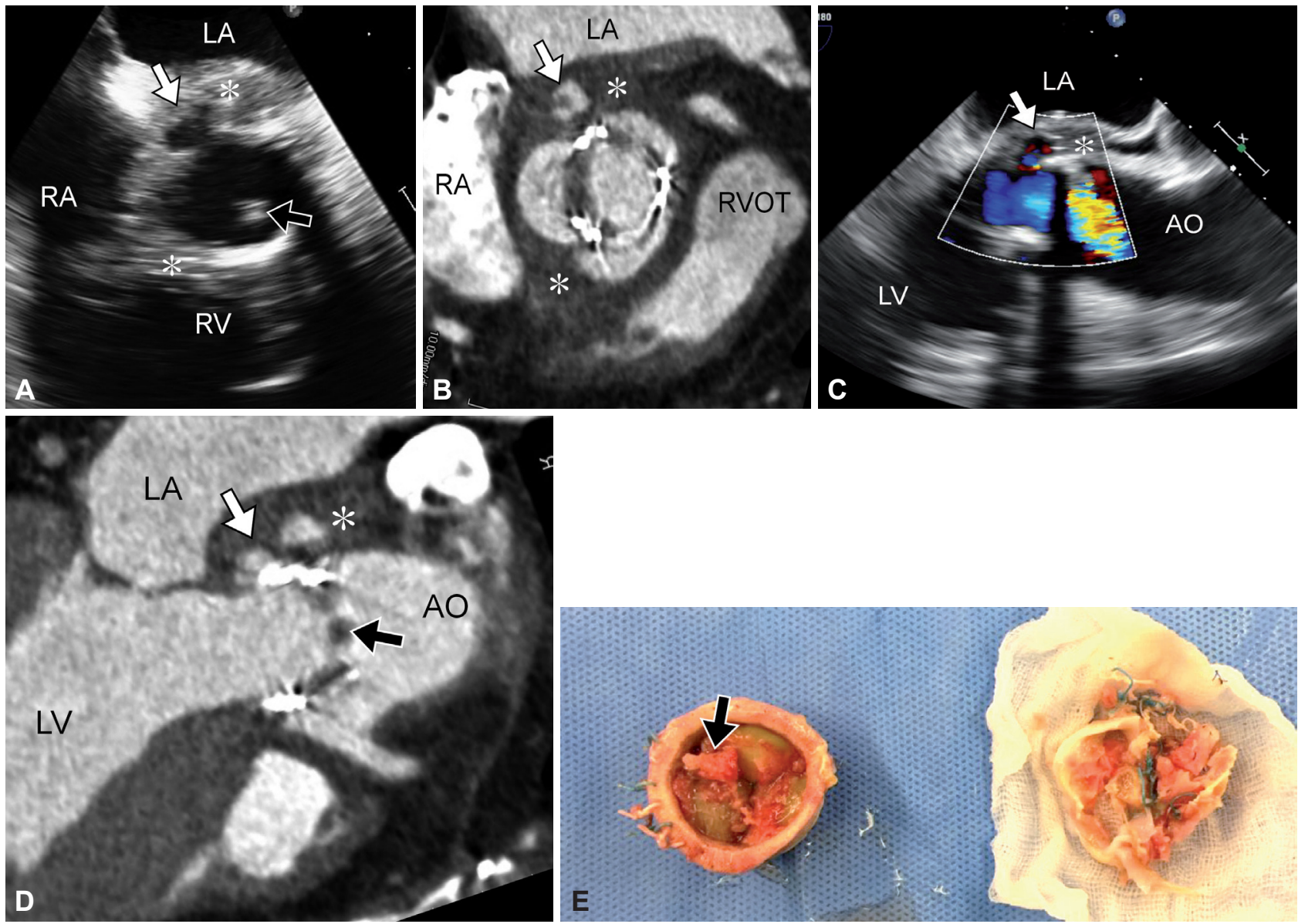

Fig. 6. Four months after surgical aortic valve replacement, a 58-year-old man with fever and chills visited the emergency department. (A) On transesophageal echocardiography, a small, low-echogenic lesion (white arrow) suggesting an abscess is visible in the thickened echogenic inflamed periaortic area (asterisk). A small movable echogenic focus (black arrow) attached to the prosthetic aortic valve is also seen. (B) Aortic valve in-plane view of computed tomography image also shows a small paravalvular abscess (arrow) and slightly increased soft tissue attenuation (asterisks) in the paravalvular area. (C) Mitral aortic intervalvular fibrosa thickening (asterisk) and internal low echogenicity (white arrow) are visible on the left ventricular outflow tract view. (D) On computed tomography, corresponding areas of inflammation (asterisk) and abscess (white arrow) involving mitral aortic intervalvular fibrosa are evident. A small, low-attenuation (black arrow) vegetation is attached to the prosthetic valve. (E) Surgical specimen revealed vegetation (black arrow) and fibrous thickening of the aortic tissue valve. AO: aorta, LA: left atrium, LV: left ventricle, RA: right atrium, RV: right ventricle, RVOT: right ventricular outflow tract.
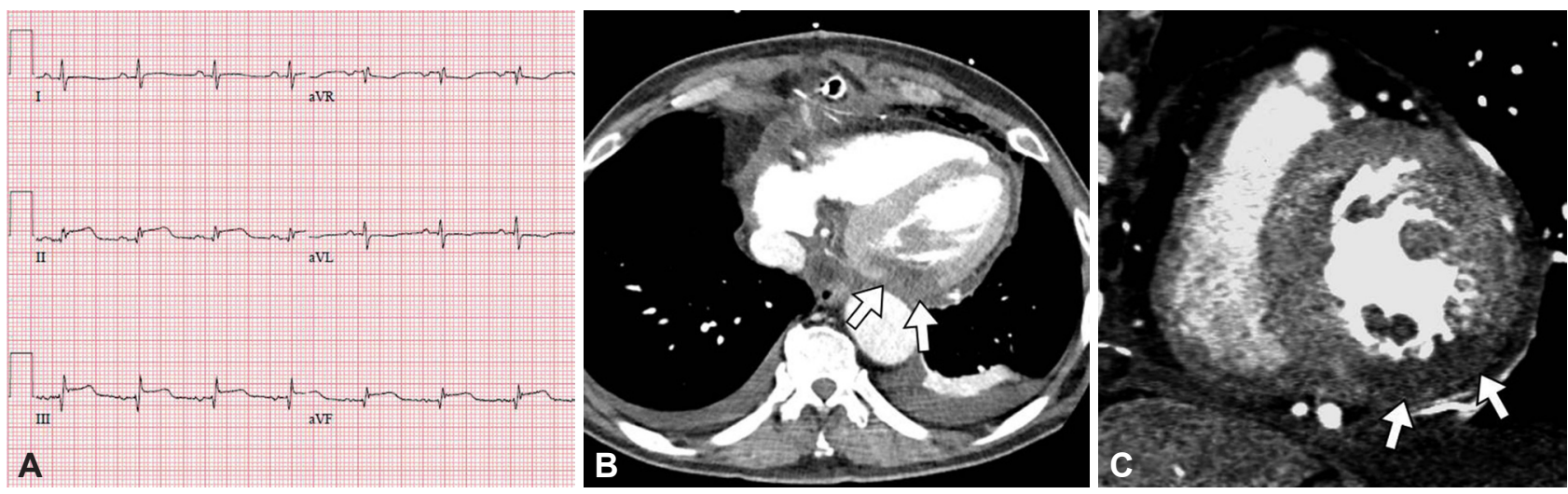

Fig. 7. A 72-year-old man who underwent a Bentall operation with prosthetic aortic valve replacement 6 days earlier due to ascending aorta aneurysm with aortic regurgitation. (A) The patient presented with chest pain, and postoperative electrocardiography showed abnormal II, III, and aVF elevations suggesting acute inferior wall infarction. (B and C) On postoperative computed tomography, myocardial low-attenuation areas in the mid to basal inferior and inferolateral wall (arrows) are noted, suggesting myocardial ischemic insults. 


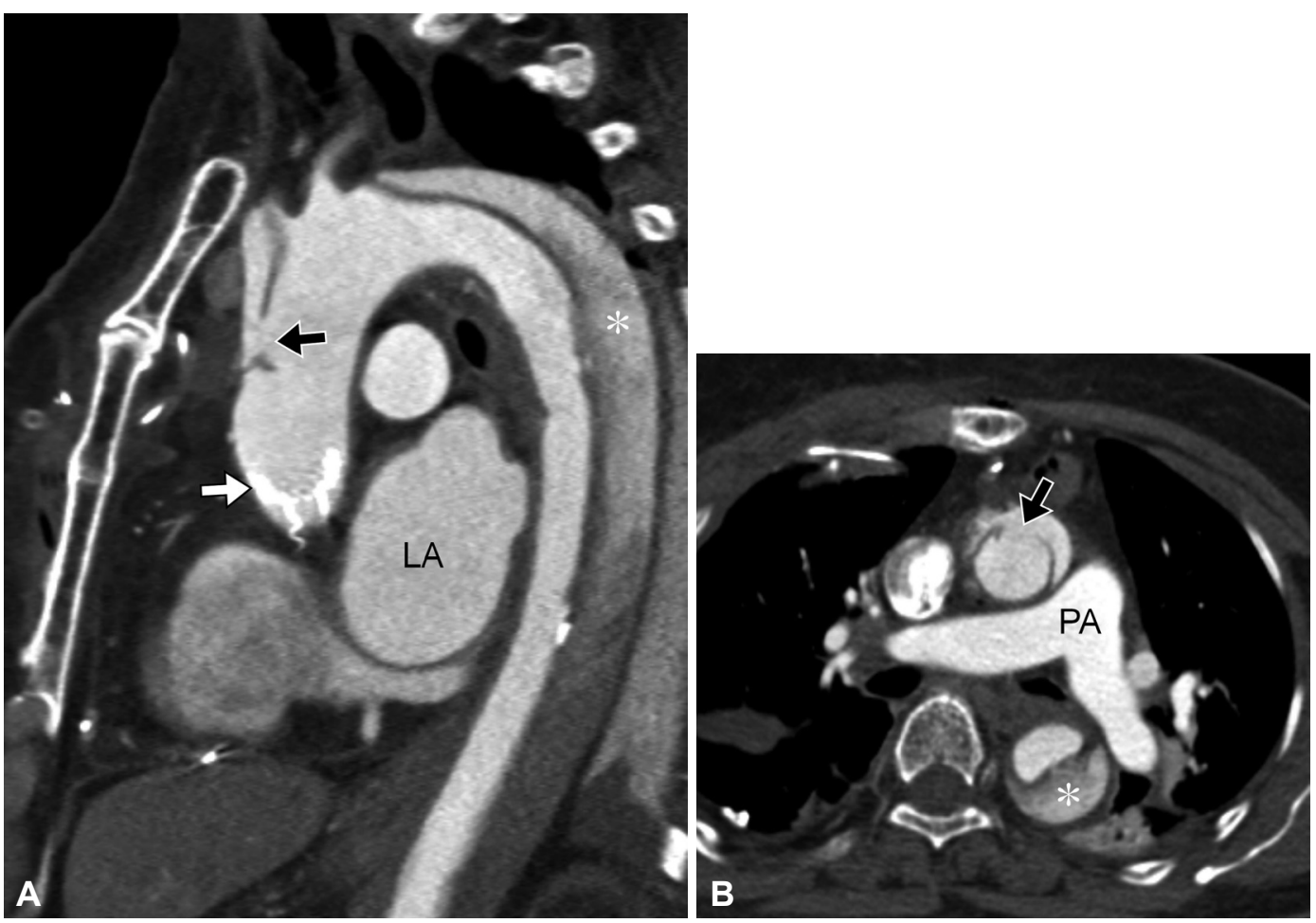

Fig. 8. A 75-year-old woman with severe aortic stenosis managed by sutureless aortic valve replacement. (A) latrogenic DeBakey type I aortic dissection is visible on a sagittal computed tomography image obtained 1 day after the operation (white arrow, mechanical valve; black arrow, entry site; asterisk, false lumen). (B) The entry site (black arrow) is located near the suture site of the ascending aorta (asterisk, false lumen). LA: left atrium, PA: pulmonary artery.

with mechanical aortic valves who are treated with anticoagulation using a vitamin $\mathrm{K}$ antagonist $[55,56]$.

Coronary artery embolism is a rare cause of myocardial infarction (Fig. 7). The risk factors for coronary artery embolism include prosthetic heart valves with subtherapeutic anticoagulation, infective endocarditis, atrial fibrillation, and atrial myxomas [57]. Most cases of coronary emboli involve the left coronary artery due to preferential flow related to aortic valve morphology [58].

\section{Aortic dissection}

Type I aortic dissection after AVR occurs in approximately $0.6 \%$ of patients within 1 month to 16 years after surgery, with a poor outcome in $50 \%$ of cases [12]. Fragility of the aortic wall, aortic regurgitation, and aortic wall thinning were identified as independent risk factors for dissection after surgery; however, previous aortic cross-clamping or cannulation or type of prosthesis were not associated factors [59]. In particular, AVR of the bicuspid valve was associated with greater incidence of sudden death and delayed acute aortic complications [60].

CT is the diagnostic choice to evaluate aortic dissection with high sensitivity, high specificity, and availability. A negative TTE is not a guaranty of missed dissection, and, considering the high mortality rate of aortic dissection, CT is necessary to evaluate the whole aorta in order to exclude disease. Even a positive TTE can be followed by CT to evaluate the involved extent and assist preoperative planning. In CT images, aortic dissection can be diagnosed by identifying an intimal flap separating the false and true lumens [61], with a reported diagnostic accuracy of 88-100\% [62] (Fig. 8). Ulcer-like projections, displaced intimal calcifications, and compression of the true lumen are additional aortic dissection findings. A lumen with a larger cross section and beak sign usually indicates a dissection-related false lumen [62].

\section{CONCLUSION}

Multidetector CT imaging is becoming popular as a diagnostic modality complementary to echocardiography. CT allows objective anatomic visualization of valvular as well as perivalvular lesions, which is useful for treatment planning, accurate diagnosis, and follow-up.

\section{Conflicts of Interest}

The authors declare that they have no conflict of interest.

\section{REFERENCES}

1. Maganti K, Rigolin VH, Sarano ME, Bonow RO. Valvular heart disease: 
diagnosis and management. Mayo Clin Proc 2010;85:483-500.

2. Mărgulescu $\mathrm{AD}$. Assessment of aortic valve disease - a clinician oriented review. World J Cardiol 2017;9:481-495.

3. Phan K, Tsai YC, Niranjan N, Bouchard D, Carrel TP, Dapunt OE, et al. Sutureless aortic valve replacement: a systematic review and meta-analysis. Ann Cardiothorac Surg 2015;4:100-111.

4. Anwar AM, Nosir YF, Alasnag M, Chamsi-Pasha H. Real time three-dimensional transesophageal echocardiography: a novel approach for the assessment of prosthetic heart valves. Echocardiography 2014;31:188-196.

5. Malik SB, Chen N, Parker RA 3rd, Hsu JY. Transthoracic echocardiography: pitfalls and limitations as delineated at cardiac CT and MR imaging. Radiographics 2017;37:383-406.

6. Ionescu A, Fraser AG, Butchart EG. Prevalence and clinical significance of incidental paraprosthetic valvar regurgitation: a prospective study using transoesophageal echocardiography. Heart 2003;89:1316-1321.

7. Hammermeister K, Sethi GK, Henderson WG, Grover FL, Oprian C, Rahimtoola SH. Outcomes 15 years after valve replacement with a mechanical versus a bioprosthetic valve: final report of the Veterans Affairs randomized trial. J Am Coll Cardiol 2000;36:1152-1158.

8. O’Rourke DJ, Palac RT, Malenka DJ, Marrin CA, Arbuckle BE, Plehn JF. Outcome of mild periprosthetic regurgitation detected by intraoperative transesophageal echocardiography. J Am Coll Cardiol 2001;38:163-166.

9. Safi AM, Kwan T, Afflu E, Al Kamme A, Salciccioli L. Paravalvular regurgitation: a rare complication following valve replacement surgery. Angiology 2000;51:479-487.

10. Suh YJ, Hong GR, Han K, Im DJ, Chang S, Hong YJ, et al. Assessment of mitral paravalvular leakage after mitral valve replacement using cardiac computed tomography: comparison with surgical findings. Circ Cardiovasc Imaging 2016;9:e004153.

11. De Cicco G, Lorusso R, Colli A, Nicolini F, Fragnito C, Grimaldi T, et al. Aortic valve periprosthetic leakage: anatomic observations and surgical results. Ann Thorac Surg 2005;79:1480-1485.

12. Pham N, Zaitoun H, Mohammed TL, DeLaPena-Almaguer E, Martinez F, Novaro GM, et al. Complications of aortic valve surgery: manifestations at CT and MR imaging. Radiographics 2012;32:1873-1892.

13. Kliger C, Eiros R, Isasti G, Einhorn B, Jelnin V, Cohen H, et al. Review of surgical prosthetic paravalvular leaks: diagnosis and catheter-based closure. Eur Heart J 2013;34:638-649.

14. Rizzoli G, Russo R, Valente S, Mazzucco A, Valfré C, Brumana T, et al. Dehiscence of aortic valve prostheses: analysis of a ten-year experience. Int J Cardiol 1984;6:207-221.

15. Fagman E, Perrotta S, Bech-Hanssen O, Flinck A, Lamm C, Olaison L, et al. ECG-gated computed tomography: a new role for patients with suspected aortic prosthetic valve endocarditis. Eur Radiol 2012;22:24072414.

16. Lázaro C, Hinojar R, Zamorano JL. Cardiac imaging in prosthetic paravalvular leaks. Cardiovasc Diagn Ther 2014;4:307-313.

17. Lesser JR, Han BK, Newell M, Schwartz RS, Pedersen W, Sorajja P. Use of cardiac CT angiography to assist in the diagnosis and treatment of aortic prosthetic paravalvular leak: a practical guide. J Cardiovasc Comput Tomogr 2015;9:159-164.

18. O'Neill AC, Martos R, Murtagh G, Ryan ER, McCreery C, Keane D, et al. Practical tips and tricks for assessing prosthetic valves and detecting paravalvular regurgitation using cardiac CT. J Cardiovasc Comput Tomogr 2014;8:323-327.

19. Koo HJ, Yang DH, Kang JW, Han K, Chung CH, Song JK, et al. Demonstration of prosthetic aortic valve dehiscence in a patient with noninfectious aortitis by multimodality imaging: findings of echocardiography and computed tomography. Circulation 2013;128:759-761.

20. Raoult D, Abbara S, Jassal DS, Kradin RL. Case records of the Massachusetts General Hospital. Case 5-2007. A 53-year-old man with a prosthetic aortic valve and recent onset of fatigue, dyspnea, weight loss, and sweats. N Engl J Med 2007;356:715-725.

21. Sorajja P, Cabalka AK, Hagler DJ, Rihal CS. Percutaneous repair of paravalvular prosthetic regurgitation: acute and 30-day outcomes in 115 pa- tients. Circ Cardiovasc Interv 2011;4:314-321.

22. Ruiz CE, Jelnin V, Kronzon I, Dudiy Y, Del Valle-Fernandez R, Einhorn $\mathrm{BN}$, et al. Clinical outcomes in patients undergoing percutaneous closure of periprosthetic paravalvular leaks. J Am Coll Cardiol 2011;58:2210-2217.

23. Barbetseas J, Nagueh SF, Pitsavos C, Toutouzas PK, Quiñones MA, Zoghbi WA. Differentiating thrombus from pannus formation in obstructed mechanical prosthetic valves: an evaluation of clinical, transthoracic and transesophageal echocardiographic parameters. J Am Coll Cardiol 1998; 32:1410-1417.

24. Roudaut R, Lafitte S, Roudaut MF, Courtault C, Perron JM, Jaïs C, et al. Fibrinolysis of mechanical prosthetic valve thrombosis: a single-center study of 127 cases. J Am Coll Cardiol 2003;41:653-658.

25. Roudaut R, Serri K, Lafitte S. Thrombosis of prosthetic heart valves: diagnosis and therapeutic considerations. Heart 2007;93:137-142.

26. Huang G, Schaff HV, Sundt TM, Rahimtoola SH. Treatment of obstructive thrombosed prosthetic heart valve. J Am Coll Cardiol 2013;62:17311736.

27. Pislaru SV, Hussain I, Pellikka PA, Maleszewski JJ, Hanna RD, Schaff HV, et al. Misconceptions, diagnostic challenges and treatment opportunities in bioprosthetic valve thrombosis: lessons from a case series. Eur J Cardiothorac Surg 2015;47:725-732.

28. Deviri E, Sareli P, Wisenbaugh T, Cronje SL. Obstruction of mechanical heart valve prostheses: clinical aspects and surgical management. J Am Coll Cardiol 1991;17:646-650.

29. Sakamoto Y, Hashimoto K, Okuyama H, Ishii S, Shingo T, Kagawa H. Prevalence of pannus formation after aortic valve replacement: clinical aspects and surgical management. J Artif Organs 2006;9:199-202.

30. Girard SE, Miller FA Jr, Orszulak TA, Mullany CJ, Montgomery S, Edwards WD, et al. Reoperation for prosthetic aortic valve obstruction in the era of echocardiography: trends in diagnostic testing and comparison with surgical findings. J Am Coll Cardiol 2001;37:579-584.

31. Ueda T, Teshima H, Fukunaga S, Aoyagi S, Tanaka H. Evaluation of prosthetic valve obstruction on electrocardiographically gated multidetectorrow computed tomography--identification of subprosthetic pannus in the aortic position. Circ J 2013;77:418-423.

32. Gündüz S, Özkan M, Kalçik M, Gürsoy OM, Astarcioğlu MA, Karakoyun $S$, et al. Sixty-four-section cardiac computed tomography in mechanical prosthetic heart valve dysfunction: thrombus or pannus. Circ Cardiovasc Imaging 2015;8:e003246.

33. Symersky P, Budde RP, de Mol BA, Prokop M. Comparison of multidetector-row computed tomography to echocardiography and fluoroscopy for evaluation of patients with mechanical prosthetic valve obstruction. Am J Cardiol 2009;104:1128-1134.

34. Tsai IC, Lin YK, Chang Y, Fu YC, Wang CC, Hsieh SR, et al. Correctness of multi-detector-row computed tomography for diagnosing mechanical prosthetic heart valve disorders using operative findings as a gold standard. Eur Radiol 2009;19:857-867.

35. Teshima H, Hayashida N, Fukunaga S, Tayama E, Kawara T, Aoyagi S, et al. Usefulness of a multidetector-row computed tomography scanner for detecting pannus formation. Ann Thorac Surg 2004;77:523-526.

36. Restrepo CS, Martinez S, Lemos DF, Washington L, McAdams HP, Vargas $\mathrm{D}$, et al. Imaging appearances of the sternum and sternoclavicular joints. Radiographics 2009;29:839-859.

37. Berdajs DA, Trampuz A, Ferrari E, Ruchat P, Hurni M, von Segesser LK. Delayed primary versus late secondary wound closure in the treatment of postsurgical sternum osteomyelitis. Interact Cardiovasc Thorac Surg 2011;12:914-918.

38. Zacharias A, Habib RH. Factors predisposing to median sternotomy complications. Deep vs superficial infection. Chest 1996;110:1173-1178.

39. Templeton PA, Fishman EK. CT evaluation of poststernotomy complications. Am J Roentgenol 1992;159:45-50.

40. Misawa Y, Fuse K, Hasegawa T. Infectious mediastinitis after cardiac operations: computed tomographic findings. Ann Thorac Surg 1998;65:622624.

41. Goodman LR, Kay HR, Teplick SK, Mundth ED. Complications of me- 
dian sternotomy: computed tomographic evaluation. Am J Roentgenol 1983;141:225-230.

42. Jolles H, Henry DA, Roberson JP, Cole TJ, Spratt JA. Mediastinitis following median sternotomy: CT findings. Radiology 1996;201:463-466.

43. Habib G, Hoen B, Tornos P, Thuny F, Prendergast B, Vilacosta I, et al. Guidelines on the prevention, diagnosis, and treatment of infective endocarditis (new version 2009): the Task Force on the Prevention, Diagnosis, and Treatment of Infective Endocarditis of the European Society of Cardiology (ESC). Endorsed by the European Society of Clinical Microbiology and Infectious Diseases (ESCMID) and the International Society of Chemotherapy (ISC) for Infection and Cancer. Eur Heart J 2009;30:23692413.

44. Perrotta S, Aljassim O, Jeppsson A, Bech-Hanssen O, Svensson G. Survival and quality of life after aortic root replacement with homografts in acute endocarditis. Ann Thorac Surg 2010;90:1862-1867.

45. Wang A, Athan E, Pappas PA, Fowler VG Jr, Olaison L, Paré C, et al. Contemporary clinical profile and outcome of prosthetic valve endocarditis. JAMA 2007;297:1354-1361.

46. Mylonakis E, Calderwood SB. Infective endocarditis in adults. N Engl J Med 2001;345:1318-1330.

47. Cahill TJ, Prendergast BD. Infective endocarditis. Lancet 2016;387:882893.

48. Piper C, Körfer R, Horstkotte D. Prosthetic valve endocarditis. Heart 2001;85:590-593.

49. Birmingham GD, Rahko PS, Ballantyne F 3rd. Improved detection of infective endocarditis with transesophageal echocardiography. Am Heart J 1992;123:774-781.

50. Feuchtner GM, Stolzmann P, Dichtl W, Schertler T, Bonatti J, Scheffel H, et al. Multislice computed tomography in infective endocarditis: comparison with transesophageal echocardiography and intraoperative findings. J Am Coll Cardiol 2009;53:436-444.

51. Koo HJ, Yang DH, Kang JW, Lee JY, Kim DH, Song JM, et al. Demonstration of infective endocarditis by cardiac CT and transoesophageal echocardiography: comparison with intra-operative findings. Eur Heart J Cardiovasc Imaging. 2017 Feb 23 [Epub]. https://doi.org/10.1093/ehjci/jex010.
52. Thuny F, Grisoli D, Cautela J, Riberi A, Raoult D, Habib G. Infective endocarditis: prevention, diagnosis, and management. Can J Cardiol 2014;30: 1046-1057.

53. Le Tourneau T, Lim V, Inamo J, Miller FA, Mahoney DW, Schaff HV, et al. Achieved anticoagulation vs prosthesis selection for mitral mechanical valve replacement: a population-based outcome study. Chest 2009;136: 1503-1513.

54. Heras M, Chesebro JH, Fuster V, Penny WJ, Grill DE, Bailey KR, et al. High risk of thromboemboli early after bioprosthetic cardiac valve replacement. J Am Coll Cardiol 1995;25:1111-1119.

55. Cannegieter SC, Rosendaal FR, Wintzen AR, van der Meer FJ, Vandenbroucke JP, Briët E. Optimal oral anticoagulant therapy in patients with mechanical heart valves. N Engl J Med 1995;333:11-17.

56. Cannegieter SC, Rosendaal FR, Briët E. Thromboembolic and bleeding complications in patients with mechanical heart valve prostheses. Circulation 1994;89:635-641.

57. Levis JT, Schultz G, Lee PC. Acute myocardial infarction due to coronary artery embolism in a patient with a tissue aortic valve replacement. Perm J 2011;15:82-86

58. Kiernan TJ, Flynn AM, Kearney P. Coronary embolism causing myocardial infarction in a patient with mechanical aortic valve prosthesis. Int J Cardiol 2006;112:e14-e16.

59. von Kodolitsch Y, Simic O, Schwartz A, Dresler C, Loose R, Staudt M, et al. Predictors of proximal aortic dissection at the time of aortic valve replacement. Circulation 1999;100(19 Suppl):II287-II294.

60. Russo CF, Mazzetti S, Garatti A, Ribera E, Milazzo A, Bruschi G, et al. Aortic complications after bicuspid aortic valve replacement: long-term results. Ann Thorac Surg 2002;74:S1773-S1776;discussion S1792-S1799.

61. Harris RD, Usselman JA, Vint VC, Warmath MA. Computerized tomographic diagnosis of aneurysms of the thoracic aorta. Comput Tomogr 1979;3:81-91.

62. LePage MA, Quint LE, Sonnad SS, Deeb GM, Williams DM. Aortic dissection: CT features that distinguish true lumen from false lumen. Am J Roentgenol 2001;177:207-211. 